\title{
Letter to the editor: Speech problems and speech delay: possible underdiagnosis of selective mutism
}

\author{
Chaya Rodrigues Pereira ${ }^{1,2}$, Judith B. M. Ensink ${ }^{1,2}$, Maretha V. de Jonge ${ }^{4,5}$, Els Wippo ${ }^{2}$, \\ Ramón J. L. Lindauer ${ }^{1,2}$, Elisabeth M. W. J. Utens s $^{1,2,3,6}$ \\ ${ }^{1}$ Department of Child and Adolescent Psychiatry, Amsterdam University, Amsterdam Public Health, Amsterdam UMC, \\ Amsterdam, The Netherlands; ${ }^{2}$ Academic Center for Child and Adolescent Psychiatry the Bascule; ${ }^{3}$ Research Institute \\ of Child Development and Education, University of Amsterdam, Amsterdam; ${ }^{4}$ Institute of Pedagogical Sciences, Leiden \\ University, Leiden; ${ }^{5}$ Department of Psychiatry, University Medical Center Utrecht, Utrecht, ${ }^{6}$ Department of Child and \\ Adolescent Psychiatry/Psychology, Erasmus Medical Center - Sophia Children's Hospital, Rotterdam, The Netherlands. \\ E-mail:l.utens@debascule.com
}

Received: 5th January 2019, Accepted: 28th January 2019

Dear editor,

With great interest, we noticed the article by Zengin-Akkus, Celen-Yoldas, Kurtipak \& Özmert concerning speech delay in young children that was published in Volume 60, number 2 of 2018 . We think that some aspects of the study warrant closer attention. ZenginAkkus et al. ${ }^{1}$ investigated speech delay in 100 children and they focused specifically on isolated speech delay, autism spectrum disorder and global developmental delay. As measures, the investigators used medical history, physical examination, head circumference and play observation. An assessment was done of developmental domains (cognitive, emotional, motor and language development).

Regarding the focus of the study, we would like to underline that possibly the diagnosis of selective mutism could have been missed. Selective mutism is a rarely occurring anxiety disorder with a prevalence of $0.2-1.9 \% .^{2,3}$ It encompasses the absence of speaking in situations where children are supposed to speak. Age of onset is typically before the age of 5 years, and it seems more frequent in girls, and bilinguals. ${ }^{4,5}$

\section{Case presentation}

$K$. is a 4-year old boy, who was referred to our clinical institution. He does not speak in school, neither to teachers nor to classmates. Sometimes he communicates non-verbally, by nodding his head to indicate yes and shaking his head to indicate no. When the teacher asks him a direct question, he usually does not respond (also not non-verbally). The teacher does not know what K.'s level is, how she can test him and if he can move forward to the next class. Because of the lack of communication, the teacher suspects autism spectrum disorder. The parents do not notice a problem, at home $K$. speaks freely. When interacting with people he does not know, e.g. in a store, $K$. sometimes communicates non-verbally. Parents think K. might be shy when he does not know someone, but they expect him to grow out of it since they were both shy as children. After the intake and diagnostic process, $K$. is classified with selective mutism and treatment is started at school.

Selective mutism is often misdiagnosed or not identified, undiagnosed, due to the fact that screening questionnaires are not cross culturally available. Often there is a knowledge gap as to the existence of this disorder. Furthermore, in clinical practice, we often see children who have received untimely treatment or lengthy inadequate treatment trajectories, aimed at other problems.

According to DSM-5 criteria, ${ }^{6}$ selective mutism should not be classified in children that are not able to speak the language required in the social situation. Nevertheless, previous studies have shown that mild language difficulties or delays are quite common in children with selective mutism and their family members ${ }^{5,7,8,9}$ Lower scores on receptive language measures have been reported. ${ }^{10,11}$ 
Zengin-Akkus, Celen-Yoldas, Kurtipak \& Özmert (2018) found that in 50\% of the cases, only impairments were found in the language domain but not in any of the other domains measured by the Denver Developmental Screening Test-II. A language disorder in these cases is likely, but anxiety to speak might have caused lower scores on the language domain in comparison to the other domains in some cases. As stated, selective mutism can be overlooked especially in young children just starting (pre) school.

Interestingly, the authors found that in this sample almost one third was raised in homes with poor linguistic environment and $80 \%$ exceeded the recommended TV watching time. Parents were provided with advice regarding reducing screen time and increasing reading and communication activities with their children, which in turn had a positive effect on language skills/development in $22 \%$. However, if selective mutism had been the cause for speech difficulties in any of these cases, it would be disadvantageous to miss opportunities for timely treatment.

Recently, Oerbeck et al. ${ }^{12}$ published findings from a study that showed that cognitive behavioral therapy is effective for the treatment of selective mutism. At present, in the Netherlands there is a trial running (Dutch Trial Registry: NTR7534) in which we treat children with selective mutism with a protocol that provides behavioral therapy. The trial design paper regarding this randomized controlled trial has recently been submitted. With this letter we aim to contribute to knowledge about selective mutism and point out that screening instruments and treatment are available and under development.

\section{REFERENCES}

1. Zengin-Akkuş P, Çelen-Yoldaş T, Kurtipek G, Özmert E. N. Speech delay in toddlers: Are they only" late talkers"?. Turk J Pediatr 2018; 60: 165-172.

2. Bergman RL, Piacentini J, McCracken JT. Prevalence and description of selective mutism in a school-based sample. J Am Acad Child Adolesc Psychiatry 2002; 41: $938-946$

3. Viana AG, Beidel DC, Rabian B. Selective mutism: A review and integration of the last 15 years. Clin Psychol Rev 2009; 29: 57-67.

4. Carbone D, Schmidt LA, Cunningham CC, et al Behavioral and socio-emotional functioning in children with selective mutism: A comparison with anxious and typically developing children across multiple informants. J Abnorm Child Psychol 2010; 38: 1057-1067.

5. Elizur Y, Perednik R. Prevalence and description of selective mutism in immigrantand native families: A controlled study. J Am Acad Child Adolesc Psychiatry 2003; 42: 1451-1459.

6. American Psychiatric Association. Diagnostic and statistical manual of mental disorders (DSM-5 ${ }^{\circledR}$ ). American Psychiatric Pub, 2013.

7. Kristensen, H. Selective mutism and comorbidity with developmental disorder/delay, anxiety disorder, and elimination disorder. J Am Acad Child Adolesc Psychiatry 2000; 39: 249-256.

8. Kolvin I, Fundudis T. Elective mute children Psychological development and background factors. J Child Psychol Psychiatry 1981; 22: 219-232.

9. Oerbeck B, Kristensen $\mathrm{H}$. Attention in selective mutism-An exploratory case-control study. J Anxiety Disord 2008; 22: 548-554.

10. Kristensen $\mathrm{H}$, Oerbeck B. Is selective mutism associated with deficits in memory span and visual memory?: An exploratory case-control study. Depress Anxiety 2006; 23: 71-76.

11. Nowakowski ME, Cunningham CE, McHolm AE, et al. Language and academic abilities in children with selective mutism. Infant Child Dev 2009; 18: 271290.

12. Oerbeck B, Overgaard K.R, Stein MB, Pripp AH, Kristensen $\mathrm{H}$. Treatment of selective mutism: a 5-year follow-up study. Eur Child Adolesc Psychiatry 2018; 27: 997-1009. 